\title{
СИСТЕМНЫЙ АНАЛИЗ КОНКУРЕНТОСПОСОБНОСТИ ЦИФРОВОГО ПРЕДПРИЯТИЯ В РАМКАХ ИНФОРМАЦИОННОЙ СРЕДЫ
}

\author{
(c) 2020 Родионов Дмитрий Григорьевич \\ доктор экономических наук, профессор, Высшая инженерно-экономическая школа \\ Санкт-Петербургский политехнический университет Петра Великого, Россия, Санкт-Петербург \\ E-mail: dmitry.rodionov@spbstu.ru
}

(c) 2020 Конников Евгений Александрович

кандидат экономических наук, доцент, Высшая инженерно-экономическая школа

Санкт-Петербургский политехнический университет Петра Великого, Россия, Санкт-Петербург

E-mail: konnikov.evgeniy@gmail.com

(c) 2020 Мугутдинов Рашид Магомедгусенович

соискатель, Высшая инженерно-экономическая школа

Санкт-Петербургский политехнический университет Петра Великого, Россия, Санкт-Петербург

Процесс становления цифровой экономики на данный момент является объективной реальностью. Данный процесс в первую очередь характеризуется двумя базовыми системно связанными процессами - глобализации и цифровизации. Одним из ключевых следствий данного процесса является трансформация традиционного предприятия в цифровое предприятие. Цифровое предприятие для потребителя существует исключительно в рамках цифровой информационной среды, что в значительной мере ограничивает возможности маркетингового взаимодействия. Таким образом, цифровое предприятие является принципиально новым субъектом экономики, значительно отличающимся от традиционного предприятия. В рамках данной статьи приводится анализ свойств формирования конкурентоспособности цифрового предприятия и предлагается адаптивный инструмент системного анализа конкурентоспособности цифрового предприятия в рамках информационной среды. Данный инструмент предполагает анализ исключительно цифрового информационного среза о предприятии, что в значительной мере повышает универсальность и адаптивность данного инструмента. Приведенный инструмент может быть полностью автоматизирован, для чего рекомендуется использовать язык программирования Python 3, так как часть этапов применения данного инструмента на данный момент уже автоматизированы библиотеками для Python 3. В рамках последующих исследования предполагается автоматизация разработанного инструментария и его спецификация для нужд представителей отдельных секторов экономики.

Ключевые слова: цифровое предприятия, информационная среда, информационный срез, конкурентоспособность, тональность, системный анализ.

На современном этапе развития общество переживает новую технологическую революцию, являющуюся следствием двух базовых общемировых взаимосвязанных процессов - глобализации и цифровизации. Процесс глобализации характеризуется последовательным сокращением границ коммуникации, как социальной, так и торговой. Следствием данного процесс является взаимоинтеграция и универсализации инструментов коммуникации, а именно:

- Укрепление английского языка в качестве международного.

- Совершенствование систем международного права.

- Развитие трансфертного ценообразования.

- Формирование международных независимых коммуникативных платформ, как для целей социальной коммуникации (социальные сети, мессенджеры, тематические форумы и иные ресурсы), так и для профессиональных (в первую очередь торговых) целей (электронные биржи, интернет-магазины и маркетплейсы и прочее).

Наиболее значимым следствием данного процесса является консолидация предпринимательских структур, выраженная в слиянии и поглощении компаний, следствием чего в свою очередь является усиление и укрупнение транснациональных корпораций (далее ТНК). Причиной данного проявления процесса глобализации 
является первичное стремление компании (существующей в условиях конкурентного рынка) к приращению экономического результата и увеличению (или стабилизации) экономической эффективности, следствием чего является необходимость оптимизации процесса потребления ресурсов и расширении спроса. В условиях бесконечного обеспечения данного стремления компания достигает масштабов, при которых это возможно исключительно при расширении объективных географических, политических и коммуникативных границ компании, что выражается в открытии очагов производства в странах с наиболее доступной ресурсной базой, и поиске спроса в странах с наиболее состоятельной экономикой. Именно данные следствия глобализации провоцируют технологическое развитие, включительно выражающееся в процессе всепроникающей цифровизации.

Процесс цифровизации современной экономики является многомерным и комплексным. Стремлении ТНК к расширению спроса и снижению издержек реализации ТРУ приводит к развитию цифровых торговых платформ, включающих в себя как информационный сайт компании, так и масштабные маркетплейсы, объединяющие множество торговых предприятий. Данный процесс провоцирует необходимость увеличения скорости и стабильности средств цифровой коммуникации, следствием чего в свою очередь является повсеместное развитие интернета, и смежных технологий. Значимый буст данного развития был обеспечен пандемией COVID-19, охватившей весь мир в 2020 году. Для целей сохранения экономических связей и обеспечения основных бизнес-процессов многие предприятия были вынуждены перейти к дистанционной форме профессионального взаимодействия, что в свою очередь значительно снизило издержки физического взаимодействия. На момент написания данной статьи пандемия COVID-19 проходит пик второй фазы, и на данный момент перспективы отказа от существующих ограничений крайне туманны. Неуклонно трансформирующаяся экономика приводит к экспоненциальному росту уровня цифровизации, следствием чего является неизбежный рост доли исключительно цифрового предпринимательства.

Цифровое предприятие (Digital Enterprise) предприятие, многомерно использующее информационные технологии в качестве конкурентного преимущества, а именно в производ- стве, маркетинге и взаимодействии с клиентами, логистике, управлении, горизонтальной профессиональной коммуникации и многом другом. Компания Acatech совместно с компаниямипартнерами разработала методику, состоящую из шести базовых этапов, которые должно пройти предприятие в рамках процесса цифровизации, а именно:

1. Компьютеризация. Данный этап подразумевает снабжение предприятия основными средствами для целей организации и управления всеми производственными этапами. Данный этапа подразумевает как приобретение новых основных средств, так и модернизацию существующих.

2. Сетевое взаимодействие. Данный этап подразумевает взаимоинтеграцию технологий, которыми обладает предприятие, в единую среду. Как правило данный этап подразумевает организацию системы «Интернет вещей» (Internet of Things), позволяющей соединить последовательно существующие производственные этапы.

3. Обозримость. Данный этап подразумевает создание виртуального двойника предприятия, позволяющего моделировать последствия изменений оптимизации производства вне физического взаимодействия.

4. Прозрачность. На данном этапе производится создание автоматизированных аналитических систем, позволяющих извлекать знания из информации, получаемой на предыдущем этапе.

5. Прогнозирование. В рамках данного этапа производится автоматизированная вероятностная оценка результатов предполагаемой производственной трансформации.

6. Адаптивность. Данный этап является завершающим, и он подразумевает автоматизацию функций, связанных с адаптацией предприятия к изменению условий внешней среды.

Осмысление сущности каждого из этапов позволяет заключить, что цифровое предприятие в значительной степени отличается от традиционного предприятия. Цифровой торговое предприятие, сущностно может даже не иметь представительства, и является исключительно системной совокупностью отдельных ресурсных компонент, объединенных бизнес-процессами. Данный факт определяет значительную дифференциацию природы экономических свойств данного принципиального нового вида предприятий. 
Как традиционное предприятие, так и цифровое, обладает множеством квантифицируемых характеристик - производственных (производительность труда, материалоемкость и т.д.), финансовых (ликвидность, рентабельность и т.д.), маркетинговых (объем спроса, характеристики воронки продаж и т.д.) и прочих. Одной из наиболее комплексных характеристик предприятия является его конкурентоспособность. Существует множество определений конкурентоспособности. Экономико-математический словарь (словарь современной экономической науки) определяет конкурентоспособность как «способность, преодолевать барьеры для входа в рынок, выступать на этом рынке, выдерживая конкуренцию других аналогичных субъектов рыночных отношений» [1]. Данное определение дифференцировано в соответствии с основными этапами развития предприятия, однако, оно не определяет ключевых свойств предприятия. Более специфическое определение дают Мазилкина Е.И. и Паничкина Г.Г. Они определяют конкурентоспособность как относительную характеристику, которая выражает степень отличия развития организации от конкурентов по степени удовлетворения своими товарами потребностей людей» [2]. Данное определение концентрирует внимание на ключевом свойстве конкурентоспособности предприятия - сравнительном характере. Именно сравнение определяет конкурентоспособность предприятия. Сравнение может в значительной мере дифференцироваться в зависимости от множества факторов, однако объект сравнения неизменен иные предприятия, ТРУ или прочие субстанции, следствием потребления которых является изменение спроса на ТРУ субъекта (предприятия). Причины данного изменения в значительной степени дифференцированы. В качестве базовых причин можно назвать - более качественное или комплексное удовлетворение потребителей (всех типов потребностей, включая информационные), сравнительно более низкие затраты ресурсов потребителей, специфика социального и иного взаимодействия производителей и потребителей и многое другое. Таким образом, конкурентоспособность является крайне многомерным понятием, отражающим не только сравнительные свойства ТРУ или предприятия, но и их системное взаимодействие. Существует множество как подходов к определению данных свойств, так и инструментов их сравнительной оценки. В качестве примера можно привести многоугольник конкурентоспособности, матрицу BCG, матрицу McKinsey, модель пяти сил Майкла Портера и многое другое. Однако, как уже было отмечено ранее, цифровое предприятие имеет принципиально иную природу, подразумевающую в первую очередь минимизацию физического взаимодействия предприятия и потребителя. Для потребителя цифровое предприятие есть ничто иное как элемент информационного потока, представленный в виде сайта в интернете или приложения. Данный факт позволяет утверждать, что конкурентами предприятия в данном случае являются иные элементы информационного потока, также лишенные большинства физических характеристик, оказывающих влияние на процесс потребления. Сознание потребителя дистанцируется и воспринимает цифровое предприятие в контексте исключительно информационной среды, что дает возможность предприятию формировать конкурентные преимущества вне контекста физического мира.

Являясь в первую очередь элементом информационной среды, цифровое предприятие сущностно не отличается от иных информационных компонент, и в сознании потребителя в первую очередь является элементом ассоциации. В рамках процесса выбора ТРУ в рамках цифровой информационной среды, потребитель получает доступ к всеобъемлющей информации разного рода, а именно:

1. Официально декларируемая информация о ТРУ. Как правило речь идет о информации, размещаемой самим предприятием в рамках официального цифрового канала распространения ТРУ.

2. Официально декларируемая информация о предприятии. В данном случае речь идет о информации, размещаемой предприятием как на своем официальном цифровом информационном ресурсе, так и информации о предприятии, размещаемой на иных цифровых информационных ресурсах, но полностью или частично контролируемой предприятием.

3. Информация, раскрывающая опыт потребления и/или специфику ТРУ. Данная информация размещается потребителями или иными лицами как на официальном цифровом информационном ресурсе предприятия, так и на иных pecyрсах, и она посвящена свойствам ТРУ, его сравнительным характеристиками и прочему. 
4. Информация, раскрывающая опыт взаимодействия с предприятием. Данная информация размещается потребителями или иными лицами как на официальном цифровом информационном ресурсе предприятия, так и на иных ресурсах, и она посвящена как специфике процесса приобретения ТРУ, так и иным аспектам взаимодействия с предприятием.

5. Сопутствующая информация о ТРУ. Данная информация может быть размещена в рамках практически любого цифрового информационного ресурса, и она раскрывает любые аспекты существования ТРУ в контексте единой информационной среды.

6. Сопутствующая информация о предприятии. Данная информация может быть размещена в рамках практически любого цифрового информационного ресурса, и она раскрывает любые аспекты существования предприятия в контексте единой информационной среды.

Потребитель, получает возможность поглотить определенный (ограничиваемый исключительно самим потребителем) информационный срез, посредствам осмысления которого в его сознании формируется сравнительный образ как ТРУ, так и предприятия. Данный образ может сравниваться в сознании потребителя:

1. С иными ТРУ. В данном случае речь идет о сравнении представления потребителя о свойствах ТРУ относительно представления потребителя о свойствах ТРУ конкурентов. Необходим отметить, что объективность данной сравнительной оценки (в рамках физического мира) может быть крайне сомнительна.

2. С иными предприятиями. В данном случае речь идет о сравнении представления потребителя о характеристиках предприятия относительно представления потребителя о характеристиках конкурентов предприятия. Данная сравнительная оценка также может быть крайне необъективна.

3. С текущим общим информационным контекстом. Потребитель существует в динамичной информационной среде, формируемой вокруг него множеством несвязанных источников информации. Информации о предприятии или ТРУ является частью данного информационного потока, и может произвольно сравниваться с теми или иными компонентами. В качестве примера можно привести моментное падение спроса на фильмы с участием Кевина Спейси, вызванное установлением факта изна- силования со стороны актера. В рамках потребления цифровой информации в сознании потенциальных потребителей установилась связь между Кевином Спейси как главным актером, так и Кевином Спейси как насильником, что экстраполировалось на фильмы, как свойство.

4. С уникальным информационным контекстом самого потребителя. Каждый потребитель уникален. Данная уникальность в первую очередь проявляется постоянной динамичной дифференциацией информационного потока, окружающего потребителя на протяжении всей его жизни. Данный поток формирует сознание потребителя и базовые эмоциональные компоненты. Таким образом, если информация о ТРУ или предприятии продуцирует негативные или сравнительно менее позитивные эмоции у потребителя, или процесс потреблении ТРУ или взаимодействия с предприятием противоречат его принципам или суждениям, конкурентоспособность ТРУ или предприятии значительно снижается.

Как отмечалось ранее, информационная среда, формирующаяся вокруг потребителя крайне дифференцированная и динамично развивающаяся. Однако, также как и потребители могут быть объединены в условные группы, в соответствии с определённым набором признаков, так и окружающая их информационная среда может быть дифференцирована и структурирована. Информационную среду, с точки зрения механики потребления информационного потока, можно представить в виде динамично меняющейся совокупности аудиовизуальных образов и потока кодированной информации. Аудиовизуальные образы отражают объективную реальность, и представляют собой фотографии, видео, картины и прочее. Данная совокупность информации отличается тем, что мозг человека привык к ее естественному потреблению и автоматически ее декодирует и осмысляет. Кодированная информация в первую очередь представляет собой совокупность текстовых данных, которые человек в первую очередь должен декодировать, а далее осмыслять. Процесс декодирования является трудоемким для сознания человека, в связи с чем в рамках выбора потребления предпочтение автоматически отдается аудиовизуальному контенту. В тоже время текстовая информации значительно менее трудоемкая в производстве и распространении, в связи с чем человек как правило соблюдает баланс в рамках 
потребления информации. Анализу может быть подвергнута любая форма информации, однако в рамках данной работы предпочтение отдается текстовой информации. Данный выбор обусловлен сравнительно более развитой инструментальной базой автоматизации процесса анализа.

Поток текстовой информации генерируется всеми субъектами информационной среды. Предприятие генерирует описание ТРУ и собственное описание, потребители генерируют отзывы и комментарии относительно как ТРУ, так и предприятия, совокупность третьи лиц генерирует отстраненную информацию, составляющую как текущий контекст, в котором существует потребитель, так и ретроспективный контекст, который сформировал потребителя в прошлом. Изучением данного типа информации занимается компьютерная лингвистика. В соответствии с существующей научной базой, можно утверждать, что данная текстовая информация может быть разделена на две базовые аналитические компоненты - содержательную и тональную [3]. Под содержательной компонентой текстовой информации стоит понимать основу данной информации, определяющую ее сущность. Именно содержательная компонента позволяет сознанию идентифицировать основные свойства ТРУ или предприятия и их сравнительное состояние. Также, надо отметить, что именно содержательная компонента позволяет определить связи с общим информационным контекстом и информационным контекстом потребителя. Под тональной компонентой в свою очередь понимается эмоциональный окрас текстовой информации. Данная компонента позволяет потребителю осмыслить ТРУ или предприятие посредствам эмпатии. Единовременное потребление как содержательной, так и тональной компоненты текстовой информации позволяет сознанию потребителя достигнуть синергетического эффекта, и в результате сформировать единое (как эмоциональное, так и рациональное) сравнительное восприятие ТРУ или предприятия, и в итоге принять решение относительно целесообразности потребления ТРУ и/или взаимодействия с предприятием. Таким образом, процесс управления конкурентоспособностью цифрового предприятия может быть сконцентрирован вокруг процессов системного и адаптивного управления содержательной и тональной компонентой ТРУ и самого себя. Первичным этапом процесса управления является оценка. В рамках данной работы рассматривается исключительно оценка выделенных компонент.

В первую очередь рассмотрит содержательную компоненту и процесс ее оценки. Текстовая информация является фрагментируемой, а базовым фрагментом выступает токен - осмысленная лингвистическая единица, представляющая собой слово (как правило в его базовой форме) или словосочетание. Именно токены и их системная последовательность осмысляется потребителем текстовой информации и сопоставляются с уже известными потребителю токенами. Последовательное осмысление токенов, их сочетаний и последовательностей формирует в сознании потребителя образ ТРУ или предприятия, представляющий из себя совокупность его свойств, шкалированных в номинальной, порядковой или количественной шкале и единовременно сравниваемых с конкурентными ТРУ или предприятиями (информацией о которых владеет потребитель), а также общим или личным контекстом. Таким образом, именно систематизированная совокупность токенов и частота их упоминаний формирует в сознании потребителя образ ТРУ или предприятия. Безусловно, возможных сочетаний токенов, извлеченных их текстовой информации о ТРУ или предприятии, и токенов, которыми владеет потребитель, бесконечное количество. Однако, токены могут быть классифицированы также как и потребители. Для каждого ТРУ или предприятия могут быть сформированы массивы токенов, отражающих тот или иной аспект содержательного восприятия объекта. В качестве примера ТРУ можно привести бытовую технику. Бытовая техника обладает определёнными потребительскими свойствами, такими как надежность, качество материалов, функциональность, энергопотребление, стоимость и многими другими. Каждое из данных свойств в сознании потребителя выражается определённым набором токенов, к примеру:

- Надежность - «не ломается», «служит долго», «ни разу не подвел», «надежный», «долговечный» и т.д.

- Функциональность - «много возможностей», «универсальный», много функций», «решает любые задачи» и т.д.

- Стоимость - «дешёвый», «стоит своих денег», «лучший за свою стоимость», «доступный» и т.д.

Приведенный пример крайне локален и не 
является всеобъемлющим, однако эффективно отражает сущность содержательной компоненты. Именно за счет моделирования системной совокупности токенов, из которых формируется информационный, предприятие в состоянии формировать у потребителя целевое восприятие ТРУ или себя. Для целей сравнительной оценки, может быть использована доля той или иной содержательной составляющей в общем информационном срезе ТРУ или предприятия. Таким образом, предприятию необходимо сформировать массивы токенов целевого восприятия и оценить степень наличия данных токенов в массиве токенов общего информационного среза. Следовательно, необходимо провести токенизацию общего информационного среза ТРУ или предприятия. Процесс токенизации является одним из базовых инструментов компьютерной лингвистики. Более подробно данный процесс рассматривался авторами данной статьи в работах $[3,4,5]$. Для целей прикладного анализа может быть предложен коэффициента содержательной конкурентоспособности, рассчитываемый следующим образом:

$$
k_{k}=\frac{\overline{k_{k}}+\frac{\sum f_{j_{i}}}{F_{i}}}{2}
$$

Где:

1. $\overline{k_{k}}-$ среднее значение коэффициента содержательной конкурентоспособности.

2. $f_{j i}$ - частота упоминаний токена $\mathrm{j}$ в элементе і информационном срезе ТРу или предприятия.

3. $F_{i}$ - общее число токенов в элементе $\mathrm{i}$ информационном срезе ТРУ или предприятия.

Токен ј является частью сформированного предприятием заранее массива токенов целевого восприятия. Безусловно, данный информационный срез лишь частично управляемый, однако за счет его постоянного анализа, предприятие получает возможность адаптироваться и адаптировать ТРУ под потребности потребителей. Также, надо отметить, что предприятие может оценивать долю сразу нескольких содержательных компонент целевого восприятия, что в последствии предполагает либо суммирование соответствующих коэффициентов, либо выделение среднего или взвешенного среднего. В рамках расчета взвешенного среднего в качестве весов может выступать условная степень значи- мости той или иной содержательной компоненты целевого восприятия. Оценив данный параметр для ТРУ или самого себя в целом, и для ТРУ конкурентов или самих конкурентов, предприятие получает возможность сравнить как данные значения, так и структуру их формирования и сформулировать выводы относительно направлений трансформации информационного среза.

Далее необходимо рассмотреть тональную компоненту и процесс ее оценки. Являясь отражением эмоционального окраса текстовой информации, тональная компонента в первую очередь формирует эмоциональную специфику восприятия ТРУ или предприятия. При этом, выдвинутые ранее тезисы относительно необходимости дефрагментации текстовой информации на токена, как элементарные ее компоненты, является справедливым и в рамках оценки тональности. Превалирование той или иной эмоции над другой в рамка информационного среза также определяется частотой упоминания тех или иных токенов. Следовательно, оценка тональности предполагает в первую очередь определение доли массива токенов, определяющих тот или иной эмоциональный окрас текста, в общем массиве токенов информационного среза. На данный момент существует множество инструментов автоматизированной оценки тональности. Для анализа текстов на английском языке рекомендуется использовать инструменты библиотеки NLTK для Python 3, в то время как для целей анализа текстов на русском языке существует всего одно эффективное и бесплатное решение - библиотека Dostoevsky, также для Python 3. Обе библиотеки позволяют квантифицировать две базовые эмоции, выражаемые в тексте и оказывающие влияние на потребителя - уровень негативности и уровень позитивности. На основе возможных соотношений данных параметром может быть оценен уровень поляризации текста, или уровень превалирования одной эмоции над другой. Однако, необходимо отметить, что эмоциональное восприятие значительно более адаптивное, и требует обязательного распределения удельного веса того или иного информационного среза. В частности, более актуальные или авторитетные элементы информационного среза оказывают значительно более сильное эмоциональное влияние на потребителя. Для целей распределения удельного веса предлагается использовать метод Фишберна, что определяет механизм расче- 
та коэффициента тональной конкурентоспособности, рассчитываемый следующим образом:

$$
k_{t}=\sum \frac{2 *\left(N-d_{i}+1\right)}{\left(N-d_{i} * N\right)} * \frac{k_{p_{i}}}{k_{n_{i}}}
$$

Где:

1. $k_{p_{i}}-$ уровень позитивной тональности в і информационном срезе ТРУ или предприятия.

2. $k_{n_{i}}$ - уровень негативной тональности в і информационном срезе ТРУ или предприятия.

3. $d_{i}$ - порядковый номер і информационного среза ТРУ или предприятия в сформированной иерархии.

4. $N$ - общее число информационных срезов ТРУ или предприятия в сформированной иерархии.

Стратегия развития предприятия также должна учитывать динамику данного коэффициента и работать с его относительным приращением. Безусловно, данный коэффициент может быть наполнен более сложными эмоциональными компонентами, такими как уровень нейтральности или неоднозначности текста, однако, именно позитивная и негативная эмоции являются наиболее полярными и универсальными.

Предлагаемые коэффициенты являются исключительно базовыми, и требуют функционального совершенствования и конкретизации для каждого отдельно предприятия. Однако, в рамках наименее специализированных рынков (к примеру рынка FMCG) данные коэффициенты могут использоваться в базовой форме. По результатам оценки конкурентоспособности ТРУ или предприятия и основных конкурентов ТРУ или предприятия необходимо провести сравнительный анализ. Для целей сравнения предлагается использовать универсальную четырехсекторную структуру, подобную матрице BCG или матрице McKinsey. В качестве оси абсцисс может быть использован коэффициент содержательной конкурентоспособности, а в качестве оси ординат - коэффициент тональной конкурентоспособности. В качестве условного разделителя для обоих коэффициентов может быть использовано значение 1 , отражающее максимально нейтральное состояние уровня тональной и содержательной конкурентоспособности ТРУ или предприятия. Данный инструмент может быть назван - «Матрица информационной конкурентоспособности цифрового предприятия». Модель данной матрицы представлена на рисунке 1.

Как можно видеть, данная матрица состоит из четырех базовых секторов, каждый из которых характеризует определенное относительное состояние ТРУ или предприятия с точки зрения конкурентоспособности. Рассмотрим отдельно каждый из секторов:

1. Сектор I. Данный сектор характеризуется крайне низким уровнем как содержательной, так и тональной конкурентоспособности. Предприятия, располагающиеся в данном секторе, являются условными «информационными изгоями». В качестве ключевых рекомендаций для предприятий данного сектора можно пред-

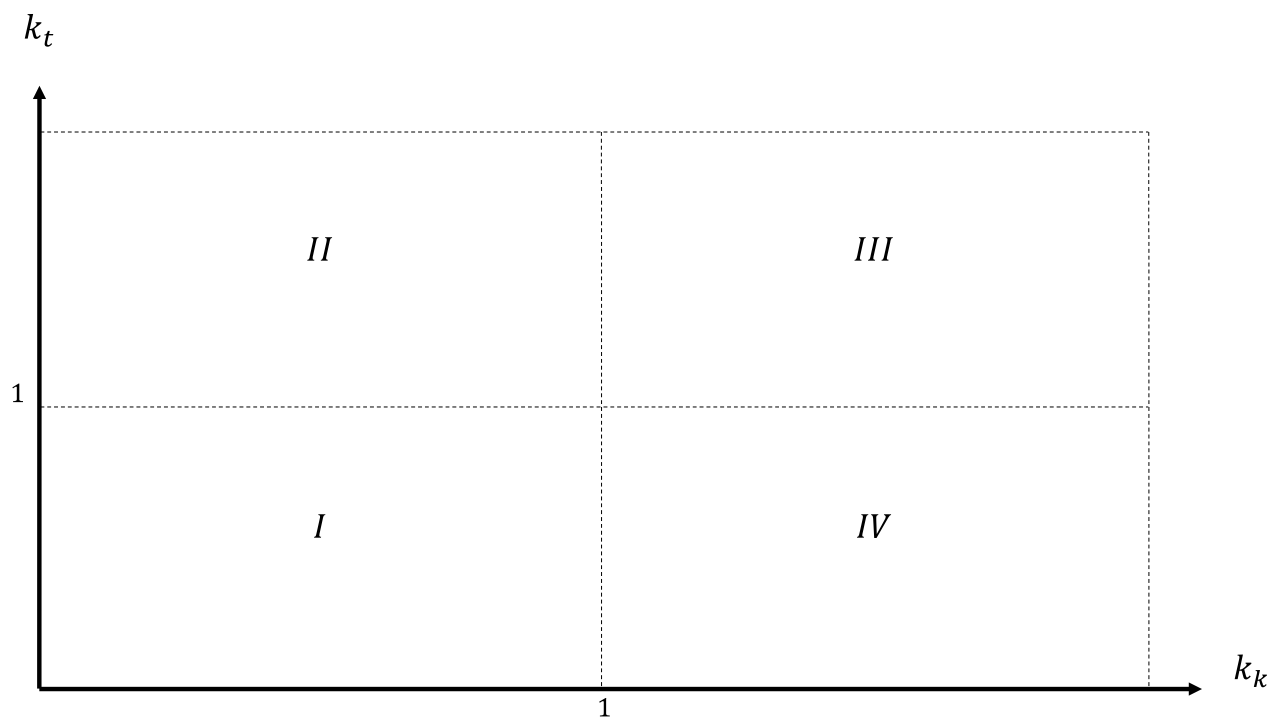

Рисунок 1. Матрица информационной конкурентоспособности цифрового предприятия 
ложить отказ от существующего бренда и развитие принципиально нового на основе существующей ресурсной базы с контролем отсутствия возможных ассоциаций.

2. Сектор II. Данный сектор характеризуется сравнительно высоким уровнем тональной конкурентоспособности при сравнительно низком уровне содержательной конкурентоспособности. Предприятия, располагающиеся в данном секторе, являются условными «народными героями». Несмотря на сравнительно низкую рациональную составляющую, информационный фон характеризуется относительно позитивной тональностью, что может быть следствием высокого уровня корреляции с существующим информационным контекстом, историческая специфика и авторитет бренда, преданное сообщество поклонников бренда и многое другое. В качестве ключевых рекомендаций для предприятий данного сектора можно предложить совершенствование объективных свойств ТРУ и последовательное укрепление в сознании потребителей данных свойств.

3. Сектор III. Данный сектор характеризуется одновременно высоким уровнем тональной и содержательной конкурентоспособности. Предприятия, располагающиеся в данном секторе, являются «лидерами рынка» в сознании потребителей. Нахождение в данном секторе говорит о значимых успехах предприятия и подтверждает эффективность его стратегии развития бренда. В качестве ключевых рекомендаций для предприятий данного сектора можно предложить проведение постоянного мониторинга состояния информационной конкурентоспособности и последовательную адаптацию в рамках трансформации потребителей.

4. Сектор IV. Данный сектор характеризуется сравнительно высоким уровнем содержательной конкурентоспособности при сравнительно низком уровне тональной конкурентоспособности. Предприятия, располагающиеся в данном секторе, являются условными «решениями для рациональных». Несмотря на объективное качество или иное объективное превосходства данных ТРУ или предприятий над конкурентами, информационный поток о данных ТРУ или предприятиях исключительно нейтральный или негативный. Причиной данного явления может быть относительная безэмоциональность бренда, исключительная прагматичность ТРУ, низкий уровень известности и многое другое. В качестве ключевых рекомендаций для предприятий данного сектора можно предложить идентификацию уникальных информационных свойств ТРУ, интеграцию в актуальный информационный контекст и, в целом, инвестиции в маркетинг.

По результатам построения матрицы информационной конкурентоспособности предприятию необходимо идентифицировать свое положение относительно конкурентов, определить целевой сектор и разработать стратегию информационной трансформации. Приведенный инструмент может быть полностью автоматизирован, для чего рекомендуется использовать язык программирования Python 3. В рамках последующих исследования предполагается автоматизация разработанного инструментария и его спецификация для нужд представителей отдельных секторов экономики.

\section{Библиографический список}

1. Экономико-математический словарь: Словарь современной экономической науки.- М.: Дело. Л. И. Лопатников. 2003.

2. Мазилкина Е.И., Паничкина Г.Г. Управление конкурентоспособностью Издательство: Омега-Л Год: 2009 , С. 27.

3. Седякина А.А., Конников Е.А. Методика оценки совместимости научно-исследовательского коллектива // Экономические науки. 2020. № 188. С. 77-87.

4. Конников Е.А., Терентьева Д. А., Конникова О.А. Анализ уровня устойчивого потребления в контексте цифровой информационной среды // Экономические науки. 2020. № 192. С. 114-125.

5. Родионов Д.Г., Ялымов С. В., Конников Е. А. Влияние информационной среды на субъекты малого и среднего предпринимательства // Экономические науки. 2020. № 189. С. 86-91.

6. Конников Е.А., Погребова О.А. Маркетинг некропроизводств // Вестник факультета управления СПбГЭУ. 2017. № 1-1. С. 277-280. 